\title{
Research Effectiveness: Some Introductory Thoughts to Share
}

\author{
Gargi Nag1, Laxmiram Gope ${ }^{2 *}$ and Gouri Sankar $\mathrm{Nag}^{3}$
}

${ }^{1}$ Department of History, City College, Kolkata, West Bengal, India

${ }^{2}$ Department of Education, Sidho- Kanho-Birsha University, Purulia, West Bengal, India

${ }^{3}$ Department of Political Science, Sidho- Kanho-Birsha University, Purulia, West Bengal, India

*Corresponding author: laxmiram.1122@gmail.com

Received: 28-02-2020 Revised: 17-06-2020 Accepted: 22-07-2020

\begin{abstract}
Research is not only fact finding endeavour but research is a comprehensive, creative and continuous process which establishes true connectivity among various phenomena. It also helps us to generate new strategies of knowledge creation and advancement of knowledge that lead us to overcome a problem through rigorous inquiry. As such our common goal is that we must conduct an effective research in pursuit of original research works. Through this study the authors being experienced practitioners of research intend to suggest and share snippets of their thought regarding some important criterion of effective research. Obviously the writers have culled inputs both from primary as well as secondary sources. But basically this writing is the result of their collective thought exercise and the root of their views lay grounded in every day academic situation and interactions with host of young researchers.
\end{abstract}

Keywords: Research, Research effectiveness, Knowledge, Trans-disciplinary, research design

Let us begin with a positive note to assure the readers that here our humble mission is to engage with students and young scholars of various social science disciplines in order to share our thoughts about what we perceive to be research effectiveness. It is pertinent therefore to add a rider at the outset that our purpose would be to offer preliminary guidance without foregrounding any fixed idea or even our past experience lest it might affect to shape present experience and thereby, might 'limit the capacity for future experience' (Seale, 2008: 21). Rather, like C. Wright Mills we believe and subscribe to the broader view that allows individual's 'intellectual craftsmanship'. In other words, research springs from a "crossroads of life experience, professional activities, and way of work" (Seale, 21). So, primarily what seems important is research curiosity and conscious thinking and probing to ask questions and answering them. So, how the researcher is thinking is very important at the first instance. It is because thinking is the stimulus by which one can envelop things into a format, sort of an inventory so that it could help us to arrange the essentials into a correlated gamut functioning like a 'working model'. It does not, however, mean thinking alone, rather carefully calibrated organized thought is the key to successful social science research.

Before we delve deeper we must also note that doing a research plan involves a great deal of personal devotion and ingenuity. It is very important that the work produces satisfaction in the mind of the researcher. Unless s/he identifies with the research work already undertaken or to be initiated and feels the work to be meaningful in the sense of value addition to the existing epistemological system, it is likely to lead to a mechanical exercise or in case of too much professional consideration propelling a research work unfortunately it might

How to cite this article: Nag, G., Gope, L. and Nag, G.S. (2020). Research Effectiveness: Some Introductory Thoughts to Share. Educational Quest: An Int. J. Edu. Appl. Soc. Sci., 11(2): 75-81.

Source of Support: None; Conflict of Interest: None (c) $\odot$ 
entail uncalled for rashness resulting in what is known as 'cutting the corner'. So, time and devotion are often intertwined although the challenge of immersing oneself in social science research leads us to acquaint and reconcile ourselves with a large pool of interconnected subjects creating prima facie a vortex of intriguing and interesting chain.

The question that we set to ourselves is what does effective research mean and why it is essential for research? Before we enter to explore the topic it won't be out of place to note that any research is valid when its conclusion is logically tenable and properly structured and organized, and research results or finding followed from the design is arrived with methodical and systematic effort that establishes a causal relation or adds to existing knowledge as something new or reaffirmed. It may or may not have policy implications or simply based on a theoretical framework wellelaborated. Therefore, research effectiveness is how systematically the study is conducted and cogently explained, hence going beyond quantitative vis-àvis qualitative research debate on strategy. Alan Bryman is absolutely correct in his assertion that "Methods are probably much more autonomous than many commentators (particularly those who espouse the epistemological versions of the debate) acknowledge" (Bryman, 2016: 125). So, it is largely 'the investigator's judgements regarding the technical viability of method in relation to a particular problem which would be important for making a research valid and reliable.

So, often what we do is to point to the tendency to associate particular methods with particular epistemological positions, but we cannot recommend it as 'a prescriptive view of how a research ought to be done'. In that sense it is difficult and arduous journey to explore the basic criterion and conditions for research effectiveness as guidance for energetic bubbling minds. Still from the point of view of novice initiators we cannot deny the importance of having instrumental support to facilitate learning some tidbits of this interesting but immensely challenging field of social science research universe.

Undoubtedly, research effectiveness is an umbrella term. It has many dimensions or wings, and which is highly dynamic and continuously evolving in terms of ever expanding ideas and notions being added to it by newer experiences in course of doing research work and contributions of the researchers not only from across the world but also from multiple disciplines and trans-disciplinary works, workshops, studies, webinars etc. So, it is quite a thought-provoking discourse in which we take recourse to both 'objectivity' or fact as-it-is, that we draw upon and also when we proceed towards interpretation or what we call 'Hermeneutics' to make sense out of collected data, not data-collection alone. Obviously, therefore, the entire process of research is an interesting journey from one level of understanding to the next higher one, thus navigating towards greater depth to bring out what we often get in terms of fine-tuned insights to enrich and embellish our research work, making it stand rigorously, distinctly with prolific articulation and execution of the entire plan. So, research effectiveness is a value concept, which does not have any constant or fixed reality. Depending upon the subject or area of study, research effectiveness may depend on collaborative and cooperative exercise or approach amongst a group of researchers, interinstitutional or inter-departmental efforts expended towards cross-fertilization and consultation with other related stakeholders. Actually research effectiveness is integrated and comprehensive process which ensures total quality of the research report. It is only possible when the research work creates an awakening like scientific spirit of enquiry. So, it is training of mind rather than an end-state which keeps on improving and thereby always fosters the quality output of the research process. So, the comprehensiveness of research process is important which denotes both horizontal stretching with comparative dimension as well as vertical depth of study. It depends on the capacity and capability of the researcher, and time, place or situation available to him/her. Thus, we can say research effectiveness is largely perceptive and often it is the function of conscious choice or approach adopted by researchers.

Now the question arises as to what we choose and why. For example, if we try to conduct a survey type of research among the slums in a locality or to find out the real situation of child labour in a city and district market, we have to justify it why we undertake such a work because we cannot defend it by saying that it is our commitment to improve the condition of the reality or our agenda is to bring 
about social change. In that case as academicians what we can do is to try toup hold the real situation and how the situation under review gets constructed in 'processual terms', thereby drawing attention of the policy makers and civil society organizations to help form social awareness. Thus putting the research to the wider social context itself creates ground for problem solving as well as it might create an introspection for negotiation because the issue as revealed by the research does not exist out there as external problem. Rather it unravels how 'socially constructed' it is. In that sense, research effectiveness does not only mean that researchers will engage only with empirical reality of the community and its working conditions that often acts as a key to develop in-depth understanding of the real world phenomena (involving the perspective of phenomenology, interactionism, participation etc), but it also incorporates the aspect of research communication and publication with ability to apply acquired findings or theoretical knowledge in practical problem solving.

Researchers might tend to think that 'quantitative data are more persuasive and hence more likely to gain the support of policy makers'. Whatsoever, it is important for 'explaining work even to non-experts' also (Bosch 2018). Because often the tendency might lead researchers to work esoterically, or make use of various complex statistical models and methods to analyze the data such as nonlinear models or Treebased methods. No doubt, therefore researchers' perspective is considered authoritative because s/he not only carries out the research but also broadly such researches and their findings are to contribute to 'a better and more rational world' and thus, these become carrier of 'national progress towards a knowledge society' (Patel, 2016: 239). By invoking Peter Drucker, Professor Pravin J Patel posits that knowledge society means "a society characterized by transition from an economy based on material goods to one based on knowledge".

Since in such a society workers are 'knowledge workers' it becomes imperative to invest in research while the common goal of research works is to generate/create new strategy for knowledge creation/affirmation. Thus, research is increasingly driving us towards knowledge intensive society in which knowledge explosion due to 'constant advent of new knowledge and advanced technology' require us to continuously update our knowledge. In other words, research effectiveness is impossible without getting oneself acquainted and acclimatized with a good number of journals, papers and several other 'new channels such as conference proceedings, open access archives and internet' which are expanding and proliferating academic disciplines and also contributing to "the qualitative aspect of the knowledge explosion" "due to escalating disciplinary, interdisciplinary and trans-disciplinary research, several new disciplines, sub-disciplines and inter-disciplines" (Patel, 2016: 242).

Although by nature research effectiveness happens to be a qualitative category, and as such it is perceptive and associated with ideographic reasoning put to explore a 'case' or some trends/ patterns that differ from others, it seems possible to hit the nails on some common features of research effectiveness which are basic for all of us - teachers, policy makers and administrators. Therefore, we can say research effectiveness is sort of strategic plan which helps us to lead forth or to crack problems with effective research process. Data becomes meaningful when magnitude is added to it. Qualitative analysis adds value to quantitative data. Of course, researcher can use the available SPSS package also for analysis of data. In such case of data analysis and presentation metrics is very important. It creates a new theoretical framework.

\section{Quality indicators of research effectiveness include:}

\section{Research effectiveness is a comprehensive and integrated term:}

(a) It's already said that research effectiveness is an umbrella term. It is associated with planning, training, preparing and executing research. These sub-categories which are mainly integrated elements of scientific inquiry and methodical and upfront approach of researchers also simultaneously encompass aspects like trendy topic, research ethic, inquisitive mind,capacity to use technology and concrete vision to look into future and deep insight regarding life, social world and the changing reality at large. It is our deep realization regarding research effectiveness that it is performance-oriented and to be informed by theoretical underpinnings. In short, it is 'method-theory relationship' and effective research cannot move forward without putting theory at the heart of the framework to arrange data. 
(b) Research effectiveness is based on strong thread of professionalism and research competence and enthusiasm of a researcher. $S /$ he must feel that it is an active process rather than a monotonous, 'lifeless or dull job, separated off from the real world' (Marsh: 2015, 4).

2. Research effectiveness is deeply epistemological because it always puts questions to the accuracy of data and patterns of thought from the standpoint of surmise. In other words, it tries to construct new relations and knowledge-claims based on constantly dynamic process and creative approach. So, the clash with traditional knowledge is always a probability because researchers in the changeable social field tend to take innovative outlook which might alter existing world-view or what may broaden the frame with more space for transformation. Undoubtedly, research effectiveness is about this intellectual sharpness, creative take of an individual researcher or a dedicated team, their efforts for nuanced study.

3. Research effectiveness depend upon research design: Research design is just like a blueprint which the architect prepares with great depth, dexterity and ingenuity before the construction of a building structure. Strong, scientific, systematic research design itself is a precondition for successful research. Generally the term 'design' means 'drawing an outline' or planning or arranging details. Thus we can say 'research design' is planning a strategy for conducting research. It plans as to what is to be observed, how it to be observed, when/where it is to be observed, why it is to be observed, how to record observation, how to analyze the data and how to generalize. But the endeavour of research design has undergone remarkable change. In this regard 'paradigm shift' can be said to have taken place in the context of design. Generally $21^{\text {st }}$ research design based on the flexibility and changeability. Therefore, if we really want to conduct an effective research study at first we should be aware about present day research design which basically promotes the mixed method or triangulation as research design.

4. Trans-disciplinary approach: Twenty first century is an age of sweeping change taking place which require viewing the world not only in terms of as it is traditionally structured but also how changes are taking place and how the social groups are engaged and affected by the turn of the situation which require eliciting phenomenological data. So, capturing the social, political, economic and cultural aspects becomes important and it leads us towards multi-method and multidisciplinary approach. It means the application of research methodology is much broader and critical than the past and in this border sense it becomes trans-disciplinary in orientation. The prefix 'trans' indicates transdisciplinary concerns which are, at once, between the disciplines, across different disciplines, and beyond each individual discipline. Its goal is understanding of the present world, of which one of the imperatives is the overarching unity of knowledge. The main theme of trans-disciplinary research is to bridge the gap in between subjects and to focus on the practicability on social and environmental issues. The main advantage of transdisciplinary research is utilitarian in that it means to find out the practicability within the theory and develop the unique evidence for problem solving.

5. Tools/test construction for data collection: In the process of research data is the main essential requirement. Without suitable data research process cannot begin, or simply research work is not feasible. Any scientific investigation depends on availability of data. A researcher can collect data through various means, some time through the help of standardized tools available in the market or sometime unavailability of suitable data might impel the researcher to embark on work through the help of self-made tools. Again sometime a researcher may need to adapt tools on Indian context for effective research. These data collection devices vary depending on the methodology a researcher may devise for conducting research. For example, if the methodology is descriptive survey then a researcher is supposed to acquire and accumulate data through closed-ended questionnaire and interview, but if the nature of the study is ethnographical then the researcher has to consider using participatory observation method and field note to keep records of emotional responses generating from the soil or his own self-reflection at some crucial moments which is subjective but highly insightful. At present vast use of Information and Communication Technology might also facilitate a researcher's quest for collecting data for the fulfillment of the research objectives. Suppose one can collect data through technological device in this pathetic pandemic era. 
6. Hypothesis: Hypothesis is considered as an eye of a researcher. The entire research work depends and proceeds according to hypothesis. Even hypothesis helps to generate the objective and hypothesis also helps us how we fulfill the objective. Effective or working hypothesis depends on the background knowledge of the problem. It means the formulation of hypothesis is dependent on a researcher's deep knowledge of the research problem 'which may be expressed in abstract terms initially but it should indicate how it can be investigated practically' (Walliman 2011: 282).

A null hypothesis is, in a sense, the reverse of a research hypothesis. It is, in fact, a no-effect or difference hypothesis or negation hypothesis that tends to refuse or deny what is explicitly indicated in a given research hypothesis (Singh, A. K 1996). It's said that null hypothesis $\left(\mathrm{H}_{0}\right)$ is a hypothesis which the researcher tries to disprove, reject or nullify. The 'null' often refers to the common view of something, while the alternative hypothesis is what the researcher really thinks is the cause of a phenomenon. A null hypothesis is ordinarily more useful than other hypotheses because it is exact (Garrett. H.E 2011). Hypotheses other than the null can be stated exactly: we may, for example, assert that a group which has received special training will be a definitive credit points on the average ahead of an untrained (i.e. Control) group. But it is difficult to set up such precise expectation in many experiments. For this reason, it is usually advisable to test against a null hypothesis, rather than some other.

7. Research effectiveness does not mean tailor-made for exclusive personalized work because once it is completed its findings must be shared with the members of academic community in the form of monograph or journal article and vital inputs of such research must always be included or incorporated in the policy perspective. So dissemination through publication is an important stage to make the work public and to evoke response. As Julie f. Scott writes, "Audience cannot be forgotten, as the public role of research texts is the crucial one....There are several audiences: institutional (researcher's university/ researcher's funder), policy (does research have an applied sense?), populists (does it have a populist agenda?), peers (is there a need for peer recognition), personal (for career development or personal satisfaction) and finally field subjects."
(Marsh 2015: 64-65). Therefore, every research report must be shared and publication to be circulated across the world as widely as possible. It is to be kept in mind that without publication research cannot proceed.

8. Research writing and drafting. Research writing and drafting is an important challenge of any kind of research either in social science or science. Writings must be creative as far as possible but clarity and objectivity in communication are most important. In case of reference writing must follow the convention or protocol of proper citation style. Even most profound ideas can best be put in simple language and in coherent sentences. According to Lester and Lester, 'when writing the body of the paper, one should classify, compare and analyze the issues. The key elements referred by them in their checklist are analysis, presentation and paragraphs' (Lester \& Lester 2015: 225-226).

9. Research effectiveness is intended to stimulate thought not only among students but amongst enthusiastic members of more generalized audience so that ideas of researchers could provoke dialogue to make things intelligible regarding the relationship of society, state and non-state actors. It is possible when a research is comprehensive and based on collaborative and cooperative approach, thus creating a new synergy between individuals or interinstitutional resources. For example, here we can compare two reputed journals namely the Economic and Political Weekly (EPW) and the Perspectives on Public Management and Governance (PPMG) in terms of publication of joint articles.

\section{EPW Publication}

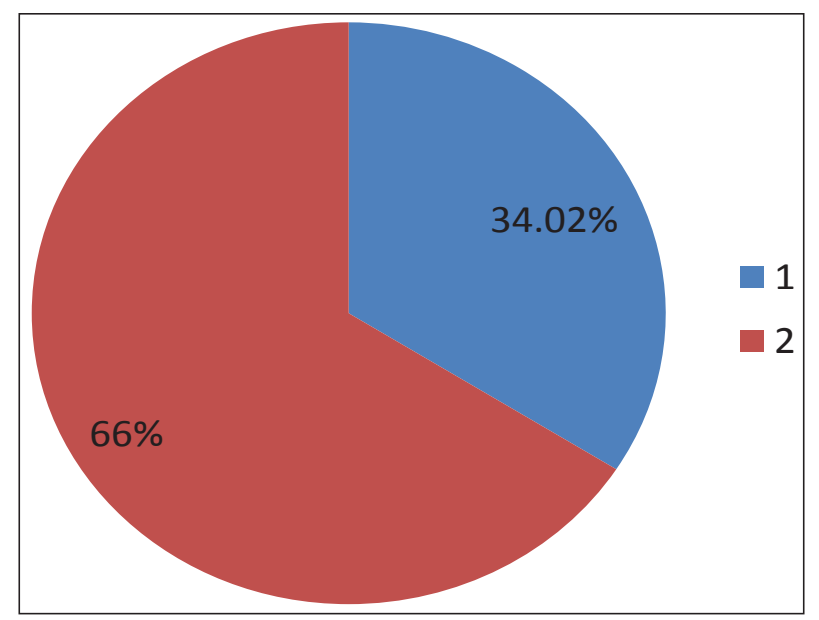

Fig. 1 
In the period from November to December 2019 out of total 97 publications 33 were joint publications. The figures reflects $34.04 \%$ publications were joint articles whereas 64 i.e. $66 \%$ articles were authored by single writer.

\section{PPMG Publication}

The Perspectives on Public Management and Governance (PPMG) is another recognized journal and through extensive survey the researchers finds following data regarding joint publication and single publication. First, the researcher put the data in the tabular form and thereafter the data were put through histogram.

Table 1

\begin{tabular}{lll}
\hline $\begin{array}{l}\text { Publication Month \& } \\
\text { Total publication }\end{array}$ & $\begin{array}{l}\text { Publication by } \\
\text { Single author }\end{array}$ & $\begin{array}{l}\text { Joint } \\
\text { publication }\end{array}$ \\
\hline March 05 & 02 & 03 \\
June 05 & 02 & 03 \\
Sept 05 & 00 & 05 \\
December 09 & 00 & 09 \\
\hline $\mathbf{2 4}$ & $\mathbf{0 4}$ & $\mathbf{2 0}$ \\
\hline
\end{tabular}

It is clear that out of 24 articles only $04(16 \%)$ is by single author while 20 (i.e. $83.33 \%$ ) articles are joint publication.

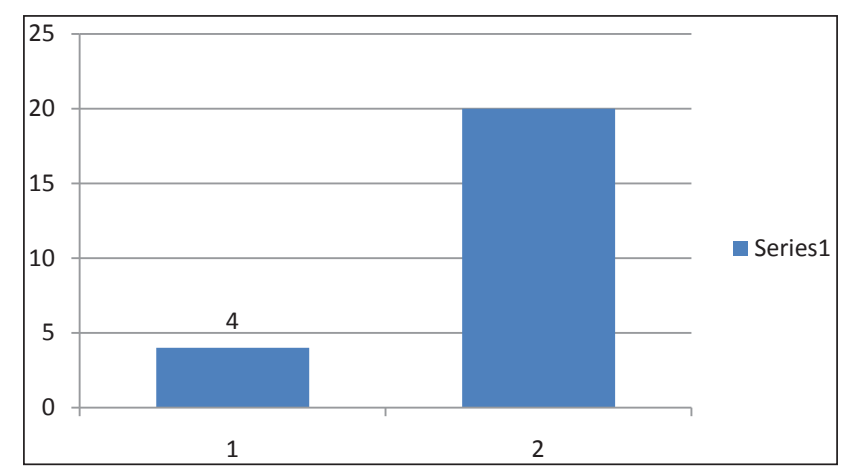

Fig. 2

10. Research effectiveness also depends upon the professional approach cum commitment of researcher. This professional approach may result from interaction and mutual negotiations among many things like specific demand of the institution that $\mathrm{s} /$ he has to meet, her/his own training, demand of the audience or pressure of commercial interest to be catered, career consideration that acts as a compulsion etc. All these factors might go into shaping the idea of trendy topics, apart from available time which researcher can afford, and policy implications of such topics, although in India "after the Nehruvian era, the connection between experts and actual policy makers grew progressively weaker" (Chakrabarti and Sanyal, 2017: 10).

11. Research effectiveness also depends upon the research ethic of a researcher. Research ethic has long historical background and it has strong normative principle to guide any researcher to conduct scientific investigation honestly and without inflicting harm on any life or violating academic integrity. Hence, specific ethical standards always make it imperative on the part of researcher to use sources with proper citation so that borrowed ideas and words could be marked out with due credit given to the original source. It not only enhances credibility of research work but also helps protect intellectual property rights of one's creativity beforehand.

According to Lester and Lester, academic citation serves other important purposes like establishing reliability. Even beyond it, cited entry in case of "non-general information" or phrase acts like documentation of borrowed ideas and words which in other words offers "A clear trail for other researchers to follow if they also want to consult the source and simultaneously as information for other researchers who might need to replicate (reproduce) the project" (2015 117). The latter is very important rather than narrowly conceived patent system. In theory, "the patent system is supposed to carefully balance the benefits of intellectual protection with another worthy goal: making access to knowledge more available." (Stiglitz, 2015:266). Although in practice, "the system has been abused" (Stiglitz 2015). So, we need also to keep in mind that despite researcher's commitment to research ethic to promote and spread knowledge, the application of research outcome might be determined by some vested interests and that cannot be prognosticated from beforehand. Still the good news lies in motivation of researchers, their fastidious outlook to keep on improving and tenacity of work and sacrifice for collective societal benefit in the long run.

\section{CONCLUSION}

Research is nothing but a creative endeavour to gain new knowledge based on sound methodological 
structure. Research effectiveness is the goal of every researcher. Yet effective research is not the sum total of a few points but it is rather a continuous pursuit of seeking to improve and validate findings based on evidence. Research assumes a social character by virtue of the very ecosystem in which a researcher works. In that sense, every research has a social purpose to fulfill with which it is integrally connected.

\section{REFERENCES}

Bosch, G. 2018. Train PhD students to be thinkers not just specialists, Retrieve https://www.nature.com/articles/ d41586-018-01853-1, viewed on May 22, 2020.

Bryman. A. 2016. Quantity and Quality in Social Research, Routledge, New Delhi.

Chakrabarti, R. and Kaushiki, S. 2017. Public Policy in India, OUP, Delhi.
Garrett, H.E. 2011. Statistics in Psychology and Education, Paragon International Publishers. 5, Ansari Road, Daryaganj, New Delhi-110-002.

Lester James, D. and James D Lester Jr. 2015. Witting Research Papers: A Complete Guide, Pearson, England.

Marsh. I. (ed). 2015. Theory and Practice in Sociology, Routledge, India.

Patel, P.J. 2016. Research Culture in Indian Universities, Social Change, Vol. 46, Issue Online article.

Seale, C. (ed) 2008. Social Research Methods, Routledge, New York.

Singh, A.K. 1996. Tests Measurements and Research Methods in Behavioral Sciences. Bharti Bhawan Publishers \& Distributors, 4271/3, Ansari Road, Daryaganj, New Delhi- 110002.

Stiglitz, J. 2015. The Great Divide, Allen Lane, India.

Walliman, N. 2011. Your Research Project, Sage, New Delhi. 
